\title{
ISELA-INIA, NUEVA VARIEDAD DE UVA DE MESA
}

\author{
Isela-INIA, a new table grape cultivar
}

Nicole Hewstone O. ${ }^{1}$, Jorge Valenzuela B. ${ }^{1}$ y Carlos Muñoz S. ${ }^{1}$

\begin{abstract}
A B S T R A C T
Isela-INIA is a mid season ripening table grape (Vitis vinifera L.) cultivar released by the Table Grape Breeding Program at the La Platina Research Centre of the National Agricultural Research Institute, Santiago, Chile. The berries are medium-sized, seedless, green, firm and flavor-full. Giberellic acid combined with girdling can be employed for thinning so as to increase berries size. It usually shows two clusters per shoot, so one cluster can be removed. Some shoulders must be removed in order to improve fruit presentation; this thinning must be done before the veraison, to avoid bruising. It ripens before 'Thompson Seedless'. Post harvest life in good conditions can last up to 45 days at $0^{\circ} \mathrm{C}$.
\end{abstract}

Key words: Vitis vinifera, table grape, new cultivar, seedless.

\section{R E S U M E N}

Isela-INIA es una variedad de uva de mesa (Vitis vinifera L.) de maduración intermedia liberada por el Programa de Mejoramiento Genético de Uva de Mesa del Centro Regional de Investigación La Platina, del Instituto de Investigaciones Agropecuarias, Santiago, Chile. Las bayas son de tamaño medio a grande, sin semilla, verdes, firmes, de un suave sabor moscatel. La variedad responde al ácido giberélico para raleo y para aumentar el tamaño de las bayas en combinación con el anillado. Normalmente produce dos racimos por brote, por lo que uno de ellos puede ser removido. Madura antes que 'Thompson Seedless'. Es necesario eliminar hombros al racimo para mejorar la calidad de postcosecha de la fruta. Este proceso debe ser realizado antes de la pinta para evitar manchado de la fruta. Puede permanecer hasta 45 días a $0^{\circ} \mathrm{C}$ en buenas condiciones de postcosecha.

Palabras clave: Vitis vinifera, uva de mesa, nueva variedad, baya sin semillas.

\footnotetext{
${ }^{1}$ Instituto de Investigaciones Agropecuarias, Centro Regional de Investigación La Platina, Casilla 33/3, Santiago, Chile. E-mail: nhewston@inia.cl *Autor para correspondencia.

Recibido: 18 de mayo de $2006 . \quad$ Aceptado: 30 de agosto de 2006.
} 


\section{ANTECEDENTES}

La variedad Isela-INIA se originó en el Instituto de Investigaciones Agropecuarias (INIA), Centro Regional de Investigación (CRI) La Platina, por un cruzamiento realizado en 1992 entre las variedades Flame Seedless ( $(+)$ y Centennial ( $\left.0^{\top}\right)$. Se seleccionó durante la temporada 1998-1999. Los primeros genotipos seleccionados se clonaron y establecieron en la Estación Experimental de Vicuña $\left(30^{\circ} 02^{\prime} \mathrm{S} ; 70^{\circ} 41^{\prime} \mathrm{W}\right)$ en 1999, y se han evaluado desde el año 2001 a la fecha. A partir del 2001 se estableció en predios privados bajo manejo comercial, en Copiapó (27 $31^{\circ} \mathrm{S}$;

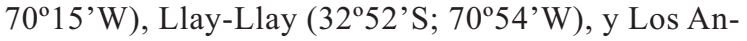
des (32 $51^{\prime}$ 'S; $\left.70^{\circ} 36^{\prime} \mathrm{W}\right)$ y en el año 2003 se estableció en Las Cabras $\left(34^{\circ} 17^{\prime} \mathrm{S}, 71^{\circ} 18^{\prime} \mathrm{W}\right)$ y Cauquenes (35 $\left.57^{\prime} \mathrm{S} ; 7^{\circ} 17^{\prime} \mathrm{W}\right)$. Desde la temporada 2003-2004 se ha podido evaluar esta selección en forma agronómica, apoyado por una evaluación de postcosecha.

\section{METODOLOGÍA}

Se utilizaron cruzamientos entre dos variedades apirenas, ya que así la población de segregantes es mayoritariamente sin semilla (Bouquet y Danglot, 1996). En las variedades de vid (Vitis vinifera L.) la ausencia de semillas es el resultado de la estenospermocarpia, es decir, hay polinización, fertilización y formación del embrión, pero este último aborta tempranamente durante su desarrollo (Kanamadi et al., 1999; Ponce et al., 2000). Una vez realizado el cruzamiento, antes del aborto del embrión, éstos se cultivaron in vitro bajo condiciones artificiales de manera de permitir su desarrollo normal (Ramming, 1990, Ponce et al., 2000; Liu et al., 2003). Los embriones se incubaron por un período variable entre 3 y 6 meses para que se desarrollaran plántulas y posteriormente plantas, las cuales se criaron y aclimataron en invernadero, previo a su plantación en el campo en espalderas distanciadas $3,5 \mathrm{~m}$ entre hileras y $1 \mathrm{~m}$ sobre la hilera.

\section{DESCRIPCIÓN DE LA VARIEDDAD}

Esta variedad produce racimos de forma cónica y densidad media de bayas. El peso promedio de los racimos, en plantas en tercer año de producción, es de $600-800 \mathrm{~g}$ si no son arreglados y de 750-1.000 g si son arreglados y tratados con ácido giberélico para la exportación. Las bayas son verdes, no tienen semilla, y de forma esférica, alargándose con los tratamientos para crecimiento con ácido giberélico. El diámetro de las bayas alcanza $20 \mathrm{~mm}$, llegando incluso hasta 24 $\mathrm{mm}$, cuando las plantas son anilladas y tratadas con ácido giberélico para crecimiento. Sin esta hormona alcanza un calibre máximo de $16 \mathrm{~mm}$. El peso promedio de la baya es de $2,5 \mathrm{~g}$ en el testigo y $5-8 \mathrm{~g}$ cuando se trata con ácido giberélico. Para crecimiento de bayas responde a dos a tres aplicaciones de 20 hasta 40 $\mathrm{mg} \mathrm{kg}^{-1}$ de ácido giberélico. Los racimos son densos cuando no se realizan aplicaciones de ácido giberélico para raleo. Responde bien a tratamientos para raleo químico de flores a inicio y $50 \%$ de floración. Posteriormente es necesario entresacar hombros para la formación del racimo comercial, ya que las bayas tratadas con ácido giberélico duplican su peso, lo que produce un racimo apretado que podría facilitar el ataque de botritis (Botrytis cinerea Pers. Ex. Fr.) en postcosecha. Las aplicaciones de ácido giberélico para raleo también tienen efecto sobre el raquis, produciendo su elongación.

La planta es muy vigorosa, con muy buena productividad y alta fertilidad de yemas. Las yemas de los sarmientos brotan en más de un $80 \%$, y la fructificación ocurre principalmente en las yemas basales, observándose normalmente dos racimos por brote, lo que hace necesario el ajuste de la carga. Los tratamientos con ácido giberélico no afectan la brotación ni la fructificación de la temporada siguiente.

La madurez de la fruta es media, madura a lo menos 10 días antes que 'Thompson Seedless'. En Los Andes alcanza la madurez la segunda quincena de enero, en Llay-Llay a principios de febrero, en Vicuña a fines de diciembre principio de enero, y en Copiapó a

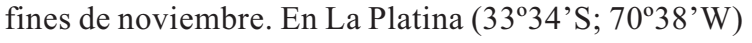
madura a fines de enero junto con 'Flame Seedless'. La variedad, en su tercera temporada de producción bajo manejo comercial, se ha manejado con 40 racimos por planta y se ha cosechado con un promedio de $16^{\circ}$ Brix y baja acidez.

\section{CARACTERÍSTICAS FITOSANITARIAS}

Esta variedad presenta un comportamiento normal frente a las plagas y enfermedades de la vid (Álvarez y Pinilla, 2000), manifestando sólo una alta susceptibilidad a oídio (Oidium tuckeri Berk.) El programa de control debe seguirse rigurosamente, requiriendo aplicaciones adicionales si las condiciones ambientales lo exigen. También se ha mostrado afectada por el complejo de organismos que producen la pudrición ácida (Penicillium sp, Aspergillius sp, Cladosporium sp y Rhizopus sp., Acetobacter spp. y levaduras).

\section{CARACTERÍSTICAS ORGANOLÉPTICAS}

La aceptabilidad se determinó mediante un panel de degustación, que indicó que la fruta de esta variedad 
tiene una buena aceptabilidad. Esta variedad presenta buena apariencia de los racimos, buen calibre de bayas y crocancia de bayas. Algunos evaluadores consideraron la selección un poco ácida al cosecharla con $15,5^{\circ}$ Brix, lo que disminuye fuertemente al alcanzar los $16^{\circ}$ Brix, por lo que es recomendable que su cosecha sea con este nivel mínimo de sólidos solubles.

\section{ZONA DE ADAPTACIÓN}

Esta variedad se estableció en toda la zona productora de uva de mesa, entre la III y VII regiones (entre $\operatorname{los} 27^{\circ}$ y $35^{\circ}$ lat. Sur). La adaptación de la variedad fue buena en todas las localidades, presentando buenas características de producción y maduración.

\section{RENDIMIENTO}

En cuanto a producción de fruta y evaluación a la cosecha, se observó que los valores de peso de racimo, bayas y calibres aumentaron a medida que madura la planta, presentando al tercer año de producción valores superiores o similares a los obtenidos en la variedad Thompson Seedless en plena producción (Cuadros 1 a 3 ).

\section{EVALUACIÓN DE POSTCOSECHA}

En la evaluación de postcosecha durante tres temporadas, esta variedad mostró que tiene buenas condiciones para almacenaje en frío, medido como desgrane, firmeza y características del escobajo (deshidratación). Alcanza 25 días en frío y 5 días a $25^{\circ} \mathrm{C}$ (shelf life) sin problemas, y llega hasta 45 días en frío más 5 días a $25^{\circ} \mathrm{C}$, siempre que el manejo del prefrío y frío sea rápido para evitar manchado de la fruta y deshidratación anticipada del escobajo. Es recomendable además realizar el embalaje en terreno (field packing).

La variedad se ha mostrado susceptible a manchado de fruta cuando la manipulación del racimo es tardía, por lo que el arreglo de racimo debe realizarse temprano en la temporada.

\section{MERCADO POTENCIAL}

Esta variedad se recomienda para el mercado de exportación. Madura antes que 'Thompson Seedless', siendo parecida en características, lo que significa que podría adelantar la producción de fruta blanca, obteniendo precios tan buenos como los mejores precios obtenidos con 'Thompson Seedless'. Además, su manejo es más fácil, alcanzando mejores calibres que 'Thompson Seedless', sin el intenso arreglo de racimos que ésta necesita.

Cuadro 1. Productividad de la var. Isela-INIA sin y con ácido giberélico (GA $A_{3}$ para crecimiento y anillado, en plantas injertadas, al tercer año de producción. Localidad Llay-Llay, temporada 2005.

Table 1. Productivity of three year Isela-INIA cv. untreated and treated with gibberellic acid (GA, and girdling, in grafted plants, at the third year of production. Llay-Llay locality, 2005 season.

\begin{tabular}{lccccc}
\hline Tratamiento & $\begin{array}{c}\text { Peso racimo } \\
(\mathbf{g})\end{array}$ & $\begin{array}{c}\text { Bayas/ } \\
\text { racimo } \\
\left(\mathbf{N}^{\mathbf{0}}\right)\end{array}$ & $\begin{array}{c}\text { Peso } \\
\text { baya } \\
\mathbf{( g )}\end{array}$ & $\begin{array}{c}\text { Diámetro } \\
\text { ecuatorial } \\
(\mathbf{m m})\end{array}$ & $\begin{array}{c}\text { Diámetro } \\
\text { polar } \\
(\mathbf{m m})\end{array}$ \\
\hline Sin GA & $903 \pm 248,6 \mathrm{~b}$ & $351 \pm 94,7 \mathrm{a}$ & $2,57 \pm 0,3 \mathrm{~b}$ & $15,3 \pm 0,84 \mathrm{~b}$ & $16,2 \pm 0,85 \mathrm{c}$ \\
$\mathrm{GA}_{3}$ & $1.144 \pm 314,3 \mathrm{a}$ & $265,17 \pm 52 \mathrm{~b}$ & $5,15 \pm 0,2 \mathrm{a}$ & $19,3 \pm 0,87 \mathrm{a}$ & $25,2 \pm 1,81 \mathrm{~b}$ \\
$\mathrm{GA}_{3}+$ anillado & $1.037,5 \pm 151,8 \mathrm{a}$ & $210,83 \pm 28 \mathrm{~b}$ & $5,18 \pm 0,2 \mathrm{a}$ & $18,9 \pm 0,76 \mathrm{a}$ & $26,1 \pm 1,28 \mathrm{a}$ \\
\hline
\end{tabular}

Promedios con igual letra no difieren entre sí según el Test de Duncan $(\mathrm{P}>0,01)$.

Cuadro 2. Productividad de la var. Isela-INIA sin y con ácido giberélico para crecimiento, en plantas injertadas, al segundo año de producción. Localidad de Los Andes. Temporada 2005.

Table 2. Productivity of Isela-INIA cv. untreated and treated with gibberellic acid (GA, in grafted plants, at second year of production. Los Andes locality, 2005 season.

\begin{tabular}{lccccc}
\hline Tratamiento & $\begin{array}{c}\text { Peso racimo } \\
(\mathbf{g})\end{array}$ & $\begin{array}{c}\text { Bayas/ } \\
\text { racimo } \\
\left(\mathbf{N}^{\mathbf{0}}\right.\end{array}$ & $\begin{array}{c}\text { Peso } \\
\text { baya } \\
\mathbf{( g )}\end{array}$ & $\begin{array}{c}\text { Diámetro } \\
\text { ecuatorial } \\
(\mathbf{m m})\end{array}$ & $\begin{array}{c}\text { Diámetro } \\
\text { polar } \\
(\mathbf{m m})\end{array}$ \\
\hline $\mathrm{Sin} \mathrm{GA}_{3}$ & $618,8 \pm 211,6 \mathrm{a}$ & $338,8 \pm 115,7 \mathrm{a}$ & $2,1 \pm 0,4 \mathrm{~b}$ & $15,6 \pm 0,82 \mathrm{~b}$ & $16,9 \pm 0,99 \mathrm{~b}$ \\
$\mathrm{GA}_{3}$ & $850 \pm 178,7 \mathrm{a}$ & $214 \pm 57,4 \mathrm{a}$ & $4,55 \pm 0,4 \mathrm{a}$ & $17,6 \pm 0,76 \mathrm{a}$ & $23 \pm 1,67 \mathrm{a}$ \\
\hline
\end{tabular}

Promedios con igual letra no difieren entre sí según el Test de Duncan $(\mathrm{P}>0,01)$. 
Cuadro 3. Comparación de la productividad de los mejores tratamientos por localidad de la var. Isela-INIA con producciones comerciales de 'Perlette' $y$ 'Thompson Seedless'.

Table 3. Productivity of the best treatment for Isela-INIA cultivar compared to 'Perlette' and 'Thompson Seedless'.

\begin{tabular}{llccccc}
\hline Variedad & Localidad & $\begin{array}{c}\text { Año } \\
\text { producción }\end{array}$ & $\begin{array}{c}\text { Racimos } \\
\left(\mathbf{N}^{\mathbf{}}\right)\end{array}$ & $\begin{array}{c}\text { Peso racimo } \\
(\mathbf{g})\end{array}$ & $\begin{array}{c}\text { Peso baya } \\
(\mathbf{g})\end{array}$ & $\begin{array}{c}\text { Calibre baya } \\
(\mathbf{m m})\end{array}$ \\
\hline Isela-INIA & Llay-Llay & 3 & $87,80 \pm 7,79$ & $1.144 \pm 314,3$ & $5,15 \pm 0,2$ & $19,3 \pm 0,87$ \\
Isela-INIA & Los Andes & 2 & $32,4 \pm 8,32$ & $850 \pm 178,7$ & $4,55 \pm 0,4$ & $17,6 \pm 0,76$ \\
Isela-INIA & Vicuña & 1 & $38,58 \pm 12,46$ & $702,3 \pm 136,7$ & $3,98 \pm 0,34$ & $18,53 \pm 0,45$ \\
Perlette & Vicuña & 6 & $39,25 \pm 22,43$ & $534,6 \pm 83,9$ & $3,2 \pm 0,2$ & $16,5 \pm 0,6$ \\
T. Seedless & El Milagro $^{1}$ & 6 & $29,80 \pm 3,96$ & $1.131,99 \pm 299,3$ & $5,91 \pm 0,4$ & $17,6 \pm 0,73$ \\
\hline
\end{tabular}

Valores \pm desviación estándar.

${ }^{1} 34^{\circ} 10^{\prime} \mathrm{S} ; 70^{\circ} 47^{\prime} \mathrm{W}$.

\section{LITERATURA CITADA}

Alvarez, M., y B. Pinilla. 2000. Enfermedades. p. 211233. In Jorge Valenzuela (ed.) Uva de mesa en Chile. Instituto de Investigaciones Agropecuarias, Santiago, Chile.

Bouquet, A., and Y. Danglot. 1996. Inheritance of seedlessness in grapevine (Vitis vinifera L.). Vitis 35:35-42.

Kanamadi V.C., P.R . Dharmatti, P. Shankaragouda-Patil, P.M., Gangadharappa, and B.C. Patil. 1999. Histological and histochemical status of embryo development in open pollinated stenospermocarpic grapes (Vitis vinifera L.) Adv. Plant Sci. Res. India 9:113-115.
Liu, S.M., S.R. Sykes, and P.R. Clingeleffer. 2003. Improved in ovulo embryo culture for stenospermocarpic grapes (Vitis vinifera L.). Aust. J. Agric. Res. 54:869-876.

Ponce, M.T., C.B. Agüero, M.T. Gregori, and R. Tizio. 2000. Factors affecting the development of stenospermic grape (Vitis vinifera) embryos cultured in vitro. Acta Hortic. 528:667-672.

Ramming, D.W. 1990. The use of embryo culture in fruit breeding. HortScience 25:339-342. 